\title{
La carpeta de aprendizaje: una innovación docente en la asignatura de Radiología y Medicina Física Especial
}

\author{
J.D. Berná, M. Reus-Pintado, J.M. Moreno-Fernández, M. Ruzafa-Martínez, M. Madrigal-De Torres
}

\begin{abstract}
Objetivo. Presentar una innovación docente desarrollada en la asignatura de Radiología y Medicina Física Especial durante el curso académico 2006-2007. Sujetos y métodos. Los alumnos realizaron una rotación durante dos semanas en los servicios de Radiodiagnóstico y Medicina Nuclear, en grupos pequeños. La metodología docente utilizada fue la carpeta de aprendizaje o portafolio. Se describen los aspectos fundamentales para la elaboración de la carpeta y de los casos clínicos, así como del sistema de evaluación. Dentro del ciclo de mejora continuada se llevó a cabo una encuesta de satisfacción. Resultados. Los resultados académicos obtenidos fueron excelentes. La mayoría de los alumnos consideraron que la asignatura fue interesante. También señalaron una valoración positiva de la rotación, de los seminarios y del sistema de evaluación. Asimismo, la opinión de los alumnos acerca de la experiencia docente realizada fue muy favorable. Conclusión. La satisfacción global de los alumnos ha sido muy alta con la metodología docente implantada en la asignatura.
\end{abstract}

Palabras clave. Aprender a aprender. Carpeta de aprendizaje. Encuesta de satisfacción. Metodología activa. Portafolio.

\section{The portfolio: an innovate educational project on} the subject of Radiology and Special Physical Medicine

Aim. To introduce a learning innovation on the subject of Radiology and Special Physical Medicine along the academic year 2006/2007. Subjects and methods. Working in small groups, students carried out a two-week rotation in the departments of Radiology and Nuclear Medicine. The portfolio was the educational methodology used. The main aspects to elaborate the portfolio, the case reports, as well as the evaluation system are described. A satisfaction survey within the continuous improvement course was performed. Results. The academic results achieved were excellent. The subject was considered interesting by most of the students. A positive evaluation about the rotation made by the students, the seminaries and the evaluation system was made. Students' opinion about the educational experience performed was also, very favourable. Conclusion. The educational methodology introduced in the subject has produced a high level of satisfaction among the students.

Key words. Active methodology. Learning to learn. Portfolio. Satisfaction survey.

\section{Introducción}

La implementación del Espacio Europeo de Educación Superior (EEES) supone la gradual convergencia de los sistemas educativos de los países miembros de la Unión Europea (UE) hacia diversos elementos comunes, tales como la adopción del modelo educativo nórdico o anglosajón y la implantación del sistema de créditos europeos o European Credits Transferibles Sistem (ECTS) [1,2].

En la coyuntura actual de convergencia con el EESS se da una gran importancia al proceso de enseñanza, aprendizaje y evaluación centrada en el alumno. Esto implica la priorización de metodologías activas que permitan a los alumnos descubrir y construir conocimientos por ellos mismos, fomentando una actitud activa y positiva hacia el aprendizaje, y de este modo proporcionar estrategias para aprender a lo largo de la vida [3-11]. Entre las metodologías activas se incluyen: la carpeta de aprendizaje o portafolios,
Facultad de Medicina. Universidad de Murcia. Hospital Universitario Virgen de la Arrixaca. El Palmar, Murcia, España.

\section{Correspondencia}

Dr. Juan D. Berná. Servicio de Radiodiagnóstico. Hospital Universitario Virgen de la Arrixaca. Ctra. Madrid-Cartagena, s/n. E-30120 El Palmar (Murcia).

\section{Fax \\ +34968219267. \\ E-mail \\ jdberna@um.es}

Agradecimientos A todos los profesionales de los servicios de Radiodiagnóstico y Medicina Nuclear del Hospital Universitario Virgen de la Arrixaca, por su colaboración en la docencia práctica de la asignatura de Radiología y Medicina Física Especial. 
el aprendizaje cooperativo, el método del caso o el sistema de aprendizaje basado en problemas.

En este contexto nos planteamos las cuestiones siguientes: ¿qué metodología se puede elegir para adaptar la asignatura a las recomendaciones de la UE? ¿Cómo conseguir que los alumnos realicen un aprendizaje autónomo, reflexivo y crítico?

La estrategia docente que se seleccionó y que responde a las cuestiones planteadas fue la carpeta de aprendizaje (CA). Se decidió implementar esta metodología en la asignatura con la finalidad de introducir una herramienta docente que favorezca el trabajo autónomo del alumno, a pesar de las dudas e incertidumbres que produce la puesta en marcha de una innovación docente. Además, un aspecto clave para elegir la CA fue que la Radiología y Medicina Física Especial es una asignatura que se caracteriza porque su docencia es exclusivamente práctica.

En este trabajo se describe la experiencia de una innovación docente desarrollada por primera vez en la asignatura de Radiología y Medicina Física Especial durante el curso académico 2006-2007, con la implantación de la carpeta de aprendizaje como un elemento de enseñanzaaprendizaje y evaluación. También se analizan los resultados de una encuesta de satisfacción.

\section{La asignatura: Radiología y Medicina Física Especial}

\section{Descripción}

Es una asignatura troncal de sexto curso de la Licenciatura de Medicina (Plan 2001) que se impartió por primera vez en el segundo cuatrimestre del curso 2006-2007, y consta de un total de cinco créditos prácticos, equivalente a 3,5 créditos europeos con una estimación del volumen de trabajo del alumno de 87,5 horas. De los 110 alumnos matriculados 93 se incluyeron en el presente estudio, y se excluyeron 17 alumnos Erasmus que no siguieron el sistema de evaluación llevado a cabo en esta experiencia docente.

\section{Guía}

El primer día del curso se realizó la presentación de la asignatura a los alumnos mediante una guía docente que incluye información sobre las prác- ticas clínicas, datos de identificación del alumno, tutor responsable, normas generales de los alumnos en las prácticas (control de asistencia y horarios), objetivos de la asignatura, recomendaciones para la realización de las actividades, así como para la elaboración de la carpeta, y los criterios de evaluación.

\section{Profesorado}

Para poder desarrollar la docencia práctica de la asignatura fue necesaria la colaboración de los especialistas de los servicios de Medicina $\mathrm{Nu}$ clear y de Radiodiagnóstico de nuestro hospital, así como de los residentes de los últimos años.

La planificación docente se llevó a cabo gracias a la excelente ayuda de estos profesionales, ya que hubiera sido imposible realizar esta labor con los profesores con los que cuentan actualmente, cuatro profesores asociados y un profesor titular, que fue el coordinador de la asignatura. También se logró que los alumnos tuvieran su tutor durante la rotación.

\section{Objetivos de la asignatura}

\section{Objetivos generales}

Con las prácticas clínicas se pretende que el alumno consiga:

- Integrar y relacionar los conocimientos teóricos adquiridos durante el presente curso y en cursos anteriores, aplicándolos en situaciones de prácticas concretas.

- Adquirir nuevas habilidades en procedimientos requeridos en los servicios de Diagnóstico por Imagen.

- Mantener una actitud responsable y ética en su actuación como médico.

\section{Objetivos específicos}

Al finalizar las prácticas clínicas el alumno será capaz de:

- Conocer el funcionamiento de los servicios de Radiodiagnóstico y Medicina Nuclear para que el alumno tenga capacidad de elegir en el posgrado estas especialidades. 
- Conocer las diferentes técnicas de imagen, sus indicaciones, contraindicaciones y riesgos.

- Seleccionar el método de imagen más adecuado ante una situación clínica determinada.

- Identificar las estructuras anatómicas normales y detectar anomalías en los diferentes estudios de imagen, así como establecer una aproximación diagnóstica razonada.

\section{Sujetos y métodos}

El aprendizaje de esta asignatura se desarrolló a través de actividades prácticas, seminarios y mediante la carpeta de aprendizaje.

\section{Actividades prácticas}

- Rotación. Los alumnos realizaron una rotación durante dos semanas por las diferentes secciones de los servicios de Radiodiagnóstico y Medicina Nuclear, del Hospital Universitario Virgen de la Arrixaca de Murcia, en grupos pequeños de 10-12 alumnos (se establecieron ocho grupos), distribuidos por parejas. Se nombró un alumno coordinador de cada uno de los grupos, y su función fue servir de puente entre el grupo y el coordinador de la asignatura. Asimismo, a cada alumno se le asignó un tutor fundamentalmente para ayudarle a elaborar los casos clínicos y resolver los problemas que le surgieran durante la rotación. El horario de las prácticas fue de 8:30 a $14 \mathrm{~h}$, de lunes a viernes. Los alumnos asistieron a las sesiones clínicas de ambos servicios. También realizaron una guardia de presencia física por parejas en el Servicio de Radiodiagnóstico de 16 a 21 horas.

- Seminarios. Durante la rotación, los alumnos recibieron seis seminarios de 1 hora de duración sobre los siguientes temas: a) fundamentos de la ecografía, indicaciones de la ecografía en urgencias, resolución de casos clínicos (dos seminarios); b) sistemática de lectura de una radiografía de tórax y presentación de casos clínicos; c) sistemática de lectura de una radiografía de abdomen y resolución de casos clínicos; d) indicaciones de la tomografía computarizada y resonancia magnética en Neurorradiología (discusión de casos clínicos), y e) técnicas, indicaciones y presentación de casos clínicos en Medicina Nuclear.

\section{La carpeta de aprendizaje}

Para motivar a los alumnos y promover su implicación se dieron las recomendaciones oportunas sobre la organización de la carpeta y los criterios de evaluación fueron expuestos con claridad al inicio. Los alumnos trabajaron con un formato de carpeta libre, realizada de forma individual y presentada en soporte de papel a las tres semanas de finalizar la rotación. También se les asignó la elaboración de dos casos clínicos, que eligieron entre los casos observados durante las actividades prácticas. De este modo se fomentó que los alumnos aprendieran por sí mismos y que asumieran un carácter activo. Hay que tener presente que no deben existir dos carpetas iguales, ya que ni el proceso de aprendizaje ni la manera de presentar ese avance son iguales a las de otro alumno. La CA es un método de evaluación que permitió al profesor conocer el proceso seguido por el alumno en su aprendizaje.

\section{Recomendaciones para la elaboración de la carpeta}

La estructuración de la carpeta es similar a la de un diario de las actividades prácticas. En este estudio la carpeta se ha concebido como una recopilación individual del trabajo que realizaron los alumnos durante las actividades prácticas: resúmenes de las sesiones clínicas, seminarios de los casos clínicos más relevantes, así como cualquier experiencia que se considera interesante. Si nos basamos en estos contenidos mínimos que representan el cuerpo o la base de la estructura de la carpeta, el alumno debe resolver las interrogantes o dudas que le surjan a través de bibliografía, libros, documentación de Internet, etc. $y$ responder a las siguientes preguntas: ¿qué caso clínico me ha planteado más dudas y debo estudiar?, ¿cómo evidenciar lo que he aprendido?

Para el desarrollo de la carpeta por parte de los alumnos se distinguen las fases siguientes: recogida de evidencias, selección, reflexión y publicación $[4,10]$. La carpeta se configuró como un instrumento que el alumno utilizó de manera continuada a lo largo de todo el proceso de prácticas, para ir señalando aquellas actividades que en su opinión eran más significativas. En la estructuración de la carpeta se incluye: un índice del diario de las actividades prácticas, así como 
de los temas desarrollados, los contenidos de apoyo y de reflexión.

En nuestra experiencia de innovación docente la carpeta reflejó el trabajo realizado por el alumno durante la rotación en el estudio de la asignatura tanto hospitalario como extrahospitalario. Asimismo, los alumnos hicieron un análisis crítico de la rotación: puntos fuertes y puntos débiles, con la finalidad de establecer medidas correctoras en un futuro. También indicaron el número global de horas extrahospitalarias que utilizaron para el estudio y la elaboración de las actividades propuestas.

\section{Elaboración de casos clínicos}

Se ha creado una página web que hemos denominado Proyectobird para la publicación de los casos clínicos elaborados por los alumnos (www. proyectobird.es). En este portal se recogen las normas que deben seguir los alumnos para la elaboración de los casos clínicos. Se trata de una base interactiva de radiodiagnóstico (BIRD).

\section{Base interactiva de radiodiagnóstico}

El proyecto BIRD (2006) es una sencilla base de casos radiológicos elaborados por los alumnos de Radiología y Medicina Física Especial de la Universidad de Murcia. En BIRD se incluyen las siguientes secciones: cabeza y cuello, tórax, mama, abdomen y musculoesquelético. El acceso a través del portal permite a los alumnos consultar este contenido, poner en práctica sus conocimientos en radiología y tomar contacto con la dinámica de trabajo del Servicio de Radiodiagnóstico y Medicina Nuclear. La resolución de cada caso clínico se basa en el avance interactivo mediante la selección de la opción correcta. Las preguntas se centran en la elección del método de imagen apropiado, en la descripción de los hallazgos radiológicos, diagnóstico diferencial y el posible diagnóstico final. La información que se expone en esta web es exclusivamente de carácter docente para los estudiantes de medicina. Además, de los casos publicados en esta web docente se seleccionaron los casos problema del examen final de la asignatura.

\section{Criterios de evaluación de la carpeta}

Los criterios de evaluación se expusieron con claridad a los diferentes grupos al inicio de la ro- tación; de esta forma, los alumnos sabían cómo y en función de qué iban a ser evaluados.

A continuación se muestran algunos de los criterios utilizados en la evaluación de la carpeta: a) presentación, estructuración y organización de la carpeta; b) relevancia de los temas seleccionados; c) reflexiones y profundización en las evidencias del proceso de aprendizaje; $d$ ) aportaciones personales, y e) nivel de implicación y coherencia.

\section{Evaluación global}

La evaluación del alumno se basó en las diferentes actividades realizadas según los criterios siguientes:

- Evaluación de la rotación del alumno: representó el 50\% de la calificación. En este apartado se incluye la comprobación de asistencia a los seminarios (obligatorios) y horas de prácticas realizadas, y la elaboración de la carpeta más la realización de dos casos clínicos según el formato establecido.

- Examen práctico basado en preguntas sobre los casos clínicos elaborados por los alumnos: representó el otro 50\% de la calificación final.

\section{Mejora continuada mediante el análisis de encuestas y resultados académicos}

Para valorar la experiencia docente se han tenido en cuenta tres indicadores: resultados académicos, resultados de una encuesta de satisfacción y comentarios libres de los alumnos acerca de la rotación recogidos en la carpeta.

\section{Encuesta de satisfacción}

Se ha realizado una encuesta similar a la del tipo Student Experience of Educational Questionnaire (SEEQ) para recoger información acerca de la experiencia desarrollada en esta asignatura. La encuesta consta de diferentes cuestiones relacionadas con el aprendizaje, la organización, la evaluación, la visión general y la carga de trabajo. Las respuestas a cuestiones como ¿ ¿ $\mathrm{L}$ La asignatura le ha parecido intelectualmente estimulante o in- 


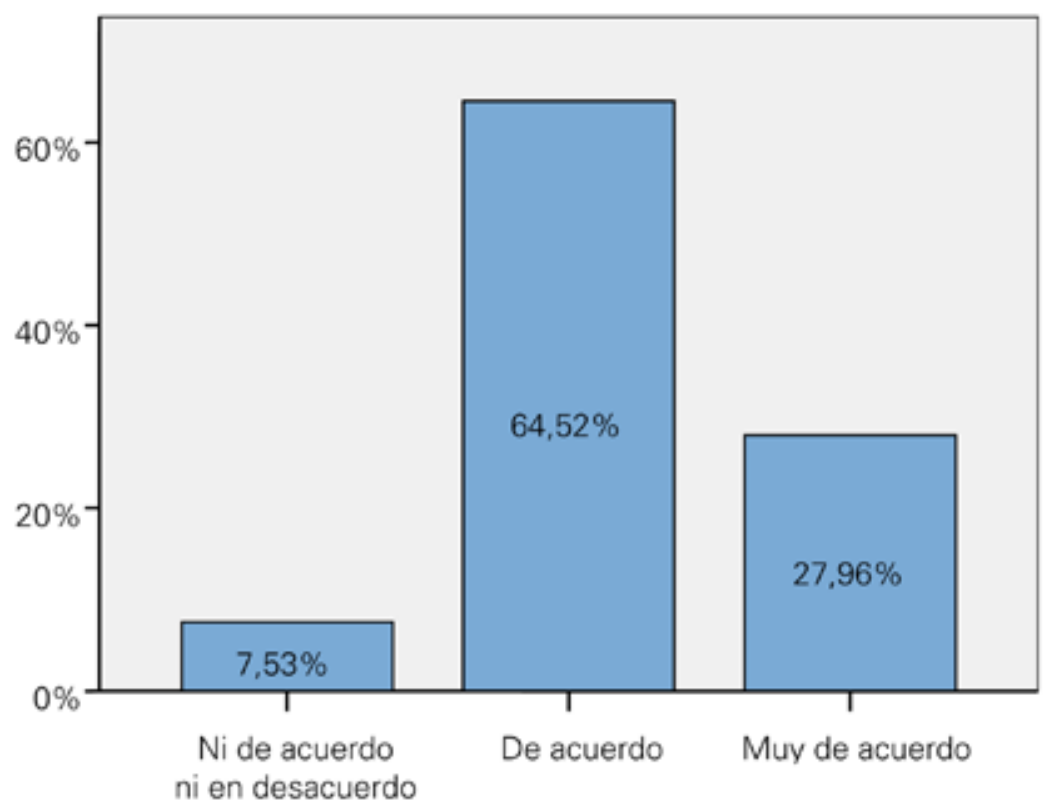

Figura 1. La asignatura me ha parecido intelectualmente estimulante o interesante..

teresante?’, ‘¿considera interesante la elaboración de los casos clínicos?' contienen una escala de 1 a 5 (1: muy en desacuerdo, 2: en desacuerdo, 3: ni de acuerdo ni en desacuerdo, 4: de acuerdo, 5: muy de acuerdo); en cuanto a las respuestas relacionadas con la valoración global de la rotación, el sistema de evaluación y los seminarios, incluyen las opciones siguientes: 1 , muy mala; 2 , mala; 3 , regular; 4 , buena, y 5 , muy buena. Cumplimentaron el cuestionario 93 alumnos (61 mujeres y 32 hombres; media de edad: 23,5 años; rango: 22-28 años) de forma voluntaria y anónima aprovechando la realización del examen práctico.

\section{Análisis estadístico de los datos}

Se realizó un análisis descriptivo de los datos calculando las frecuencias y los porcentajes para las variables cualitativas, y las medias, desviaciones estándar, o típicas, valores máximos y mínimos para las mediciones cuantitativas. A continuación se realizó un análisis bivariante, en el que se aplicó la prueba de chi al cuadrado y el test de Fisher. Se consideró significación estadística valores de $p<0.05$. Para estos cálculos se utilizó el paquete estadístico SPSS 15.

\section{Resultados}

\section{Resultados académicos}

Las calificaciones finales obtenidas por los 93 alumnos que realizaron las actividades prácticas fueron las siguientes: 2 aprobados $(2,1 \%)$, 50 notables $(53,7 \%), 35$ sobresalientes $(37,6 \%)$ y 6 matrículas de honor $(6,4 \%)$.

\section{Resultados de la encuesta de satisfacción}

Como se muestra en la figura 1, la mayoría de los alumnos consideró que la asignatura fue interesante.

Asimismo, la mayoría de los alumnos indicó que la elaboración de los casos clínicos fue interesante, el $48,3 \%$ está de acuerdo y muy de acuerdo el 39,7\%, mientras que ni de acuerdo ni en desacuerdo el 11,8\%. La valoración global de 


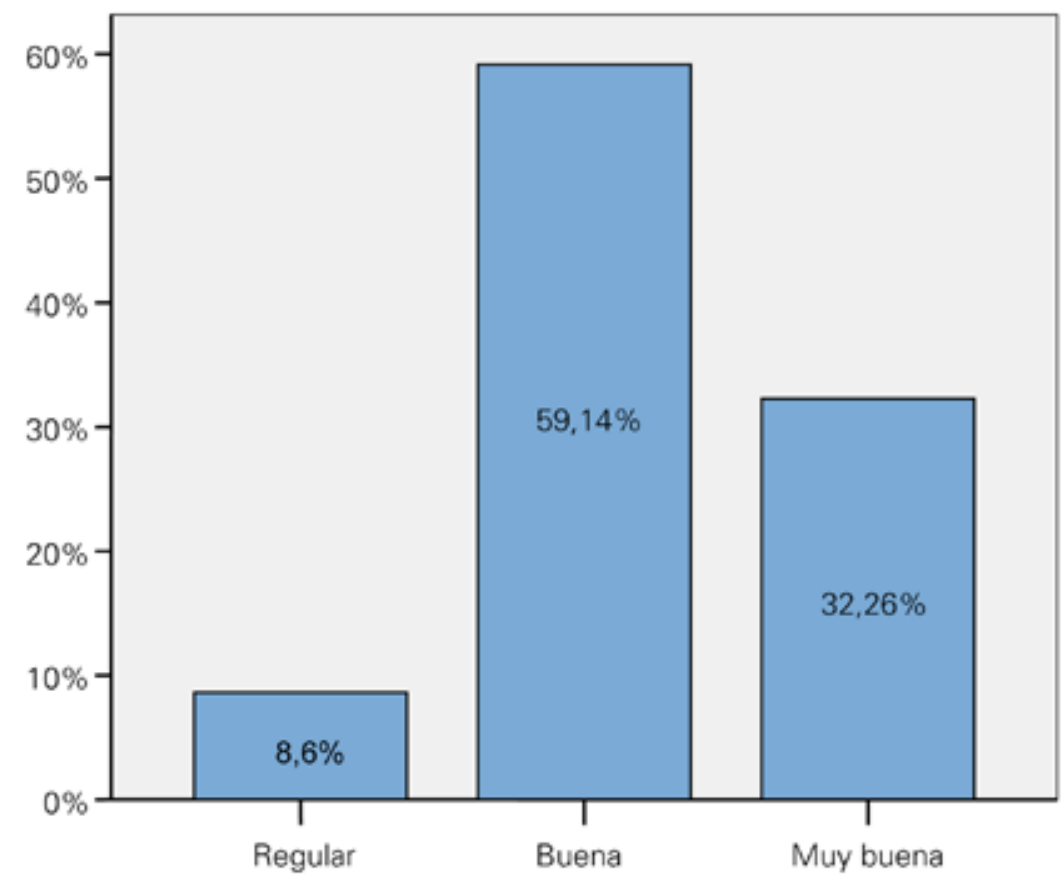

Figura 2. Valoración global de la rotación.

la rotación, así como del sistema de evaluación se muestra en las figuras 2 y 3.

En cuanto a la valoración global de los seminarios, el 79,5\% de los alumnos señaló que fue buena, y muy buena el 12,9\%, solamente el 7,5\% indicó regular. También se planteó una cuestión acerca de si el Radiodiagnóstico o la Medicina Nuclear serían su primera opción para realizar una especialidad tras el examen MIR, y la respuesta fue la siguiente: seis alumnos indicaron la especialidad de Radiodiagnóstico y dos, $\mathrm{Me}$ dicina Nuclear.

Finalmente, en relación con el número de horas de actividades extrahospitalarias relacionadas con la asignatura, los alumnos señalaron una media de 29,5 horas (desviación estándar: 22,5) con un intervalo de 10 a 120 horas.

\section{Opinión de los alumnos}

A continuación se recogen algunos de los comentarios realizados por los alumnos en sus carpetas con la finalidad de mostrar su valoración acerca de la experiencia desarrollada:
- 'La dinámica y organización de las prácticas me han parecido muy acertadas, y el trato que hemos recibido muy cordial.

- 'Quizás el único aspecto que desde un punto de vista de formación me hubiera gustado realizar es una ecografía, con la supervisión de un radiólogo, aunque sea entre los propios alumnos'.

- 'Considero que la rotación la he aprovechado y he adquirido los conocimientos básicos para poder conocer las indicaciones y las limitaciones de cada una de las técnicas radiológicas'.

- 'Han sido de las mejores prácticas de todo el curso, incluso de la carrera; completas, amenas y bien organizadas'.

- 'Es una idea muy buena impartir una asignatura de este modo porque hace que nos impliquemos más y, en definitiva, que el aprendizaje sea fructífero de verdad'.

- 'Creo que la dinámica de la asignatura está muy bien orientada y la forma de evaluación es muy motivadora, y responde al trabajo personal durante las distintas rotaciones y el tiempo dedicado. 


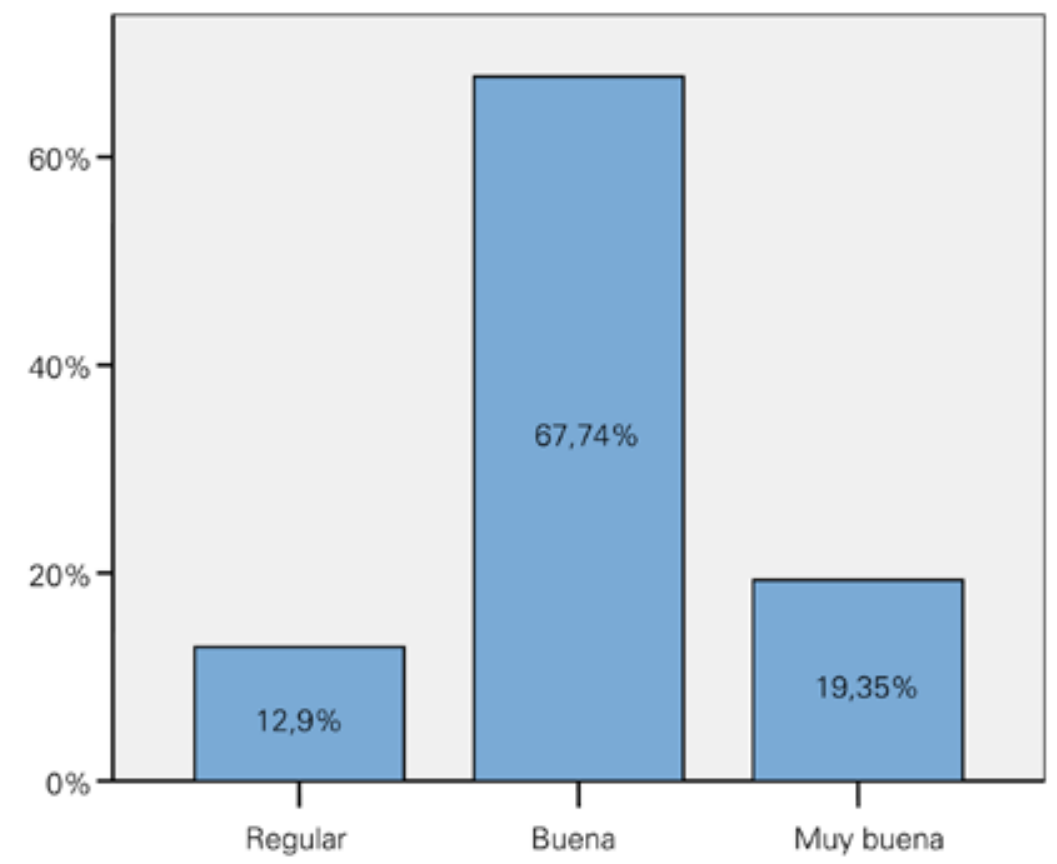

Figura 3. Valoración global del sistema de evaluación.

- 'La atención por parte de los tutores ha sido muy correcta y amable.

- 'Es de destacar la labor docente de los residentes, que nos han hecho el paso por el servicio más agradable’.

- 'Se ha conservado el significado de créditos prácticos, evitando recurrir a horas y horas de seminarios teórico-prácticos, hábito bastante común en otras asignaturas'.

- 'A pesar de ser las prácticas en las que más horas se pasaban dentro del hospital, casi nunca se tenía la sensación de perder el tiempo.

- 'La elaboración de casos clínicos ha sido algo original, y el estudio de todos ellos una forma buena de aprender'.

- 'Considero un acierto pleno enfocar de una manera esencialmente práctica la asignatura de Radiología y Medicina Física Especial, como novedad en este plan de estudios'.

- 'Esta asignatura se ha desarrollado de forma eminentemente práctica y a pesar de divergir de la norma habitual del resto de las asignaturas, nos ha permitido una formación no sólo equivalente a aquellas que poseen clases magistrales, sino que, a mi parecer, mucho mayor'.

- 'Elogiar el método didáctico impartido rompe la sintonía habitual de meros espectadores del acto médico para hacernos partícipes de él'.

\section{Discusión}

El proceso de Bolonia puede ser una 'oportunidad' de mejora de nuestras prácticas educativas. En este escenario de cambio, uno de los debates más fructíferos se ha centrado en la reflexión sobre la renovación metodológica. Con la creación del EEES se impone el diseño de nuestras asignaturas desde las competencias y la utilización de metodologías docentes que favorezcan el aprendizaje activo del estudiante, que enseñan al estudiante a aprender y a integrar la teoría con la práctica [3-11]. Uno de los aspectos más interesantes de las prácticas es permitir el análisis, la reflexión y el contraste sobre la propia actuación del alumno como profesional. Las metodologías 
elegidas son esenciales para que los alumnos adquieran conocimientos, habilidades y actitudes, y de este modo desarrollarán competencias. Una de las herramientas que más interés ha despertado en los procesos de formación es la CA o portafolio. En un trabajo reciente [11], se describe una experiencia interesante mediante la utilización del portafolio en alumnos repetidores de enfermería. También existe una propuesta de portafolio para la formación de residentes de Medicina Interna [7].

La CA o portafolio responde a dos aspectos fundamentales del proceso de enseñanza-aprendizaje, implica toda una metodología de trabajo y de estrategias docentes en la interacción entre profesor y alumno, y también es un método de evaluación [6]. Las ventajas del portafolio son que muestra una información amplia sobre el proceso de aprendizaje, promociona la autonomía del alumno y el pensamiento crítico y reflexivo, y además motiva a los alumnos, ya que se trata de un trabajo continuado. Un aspecto importante de la CA es un producto personalizado, por lo que no hay dos iguales. En cuanto a las desventajas, los alumnos se quejan de la cantidad de tiempo invertido para la elaboración de la carpeta, así como de la dificultad para tener el trabajo al día, pues este sistema no permite dejar todo el trabajo personal para el último momento. Aunque existen diferentes metodologías centradas en el alumno y en el aprendizaje, la carpeta se diferencia en las dos condiciones siguientes: contribuye y facilita la estructuración de los aprendizajes, y permite la evaluación de los aprendizajes [4].

En el presente estudio se ha utilizado la CA como innovación docente en la asignatura de Radiología y Medicina Física Especial. La colaboración de los especialistas de los servicios de Radiodiagnóstico y Medicina Nuclear de nuestro hospital, así como de los residentes, fue esencial para lograr los buenos resultados obtenidos en esta experiencia docente.

Asimismo, esta experiencia fue evaluada positivamente por el alumnado, que mostró una gran satisfacción al finalizar la elaboración de la carpeta y al realizar los casos clínicos, aunque señaló la dedicación y el esfuerzo que requiere la asignatura, como se evidencia en el promedio de horas no presenciales que le dedicaron a ésta. Sin embargo, coincidiendo con otros autores [4, $6,11]$, los alumnos mostraron cierta desorienta- ción cuando se enfrentaron a la elaboración de la carpeta debido a la libertad de creación que comporta este proceso de aprendizaje tan abierto. No obstante, hemos observado que este aspecto se minimiza cuando el alumno está informado adecuadamente de los criterios de elaboración y evaluación de la carpeta. Asimismo, al igual que otros autores $[4,11]$, resaltamos que la aplicación de esta metodología activa incrementa el tiempo de trabajo, tanto para los alumnos como para los profesores, en relación con el utilizado con métodos tradicionales.

En la experiencia docente del presente estudio se pone de manifiesto que la carpeta es un instrumento que exige al docente asumir la función de dinamizador del proceso de aprendizaje, creando entornos agradables y de confianza que favorezcan la participación del alumnado. Además, los alumnos describieron el ambiente en el que desarrollaron las actividades prácticas como cordial y participativo, y valoraron la organización como buena. Para el profesorado, la valoración de la experiencia fue positiva. Además, en este contexto se favorece la interacción profesor-alumno y la actuación del alumno como profesional. En este sentido, diversos estudios [4,6,9-11] señalan que la implementación de la CA o portafolio facilita al docente el uso de metodologías activas, en las que el alumnado, motivado y guiado por el profesorado, desarrolla un papel esencial en la adquisición del conocimiento, aprendiendo a aprender.

Por otro lado, la Radiología tiene una gran oportunidad para potenciar la docencia en el contexto del EEES, adaptando estas metodologías en nuestras asignaturas. Además, consideramos que el diagnóstico por la imagen reúne las condiciones adecuadas para estas nuevas estrategias docentes. En el presente estudio se observó que los alumnos tienen un gran desconocimiento del funcionamiento de los servicios de Radiodiagnóstico y Medicina Nuclear. Esta asignatura les ha permitido conocer ambos servicios, así como la labor que realizan los profesionales; de este modo podemos fomentar el interés del alumnado universitario por nuestras especialidades.

Finalmente, consideramos que la puesta en práctica de la CA no es una tarea fácil, ya que requiere un gran esfuerzo por parte del alumnado y del equipo docente. Sin embargo, creemos que merece la pena aceptar el reto. Destacamos que en la experiencia docente desarrollada los 
resultados obtenidos han sido muy positivos en lo que se refiere a los resultados académicos y al grado de satisfacción que mostraron los alumnos en relación con la asignatura.

\section{Bibliografía}

1. La integración del sistema universitario español en el Espacio Europeo de Educación Superior (documento marco). Madrid: MEC, 2003. URL: http://www.mec. es/universidades/eees/files/Documento_Marco.pdf [31.01.2008].

2. Consejo de Coordinación Universitaria. Propuestas metodológicas para la renovación de las metodologías educativas. Madrid: MECD, 2006. URL: http://www.mecd.es/ educa/ccuniv/html/metodologias/docu/propuesta_renovacion.pdf [31.01.2008].

3. Escayola-Maranges AM, Vila-Gangolells M. A las puertas del cambio en la formación universitaria. Revista de Educación Médica 2005; 8: 69-73.

4. Colén MT, Giné N, Imbernón F. La carpeta de aprendizaje del alumnado universitario. Barcelona: Octaedro; 2006.
5. Fernández A. Formación pedagógica y desarrollo profesional de los profesores de universidad. Análisis de las diferentes estrategias. Revista de Educación 2003; 331: 171-9.

6. Peña-González J, Ball-Vargas M, Barboza-Peña FD. Una aproximación teórica al uso del portafolio en la enseñanza, el aprendizaje y la evaluación. Revista Venezolana de Educación (Educere) 2005; 9: 599-607.

7. Propuesta de portafolio SEMI (Sociedad Española de Medicina Interna). URL: http://www.fesemi.org/ grupos/formacion/proyectos/propuesta_portafolio.doc [30.11.2007].

8. Font A. Una experiencia de autoevaluación y evaluación negociada en un contexto de aprendizaje basado en problemas (ABP). Revista de la Red Estatal de Docencia Universitaria 2003; 3: 100-12.

9. Barret H. Electronic portfolio. URL: http://electronicportfolios.org [31.01.2008].

10. Barbera E. Calificar el aprendizaje mediante la evaluación del portafolio. Revista Perspectiva Educacional 2005; 45: 69-84.

11. Vera-Cortés ML, Canalejas-Pérez MC. El portafolio como recurso de aprendizaje e instrumento de evaluación de estudiantes repetidores de enfermería. Educ Med 2007; 10: 114-20. 